\title{
Open Reviewing for Imparted Information to Effective Client Denial in the Cloud
}

\author{
M.Nanda Kishore \\ MCA., M.Tech \\ Assistant Professor, \\ Department of MCA, \\ Sri Venkateswara College of \\ Engineering and Technology, \\ Chittoor
}

\author{
S.Aasiya \\ PG Scholar, \\ Department of MCA, \\ Sri Venkateswara College of \\ Engineering and Technology \\ Chittoor
}

\author{
V.Mounika \\ PG Scholar, \\ Department of MCA, \\ Sri Venkateswara College of \\ Engineering and Technology, \\ Chittoor
}

\begin{abstract}
With information stockpiling and imparting administrations in the cloud, clients can undoubtedly change and offer information as a gathering. To guarantee imparted information uprightness can be checked openly, clients in the gathering need to process marks on all the squares in imparted information. Diverse squares in imparted information are for the most part marked by distinctive clients because of information adjustments performed by diverse clients. For security reasons, once a client is repudiated from the gathering, the squares which were beforehand marked by this denied client must be re-marked by a current client. The direct system, which permits a current client to download the comparing piece of imparted information and re-sign it amid client Disavowal, is wasteful because of the substantial size of imparted information in the cloud. In this paper, we propose a novel open evaluating instrument for the respectability of imparted information to productive client disavowal in mind. In expansion, an open verifier is constantly ready to review the uprightness of imparted information without recovering the whole information from the cloud, regardless of the fact that some piece of imparted information has been re-marked by the cloud. Additionally, our instrument has the capacity bolster cluster inspecting by checking numerous examining assignments all the while. Trial results demonstrate that our system can fundamentally enhance the effectiveness of client rejection.
\end{abstract}

GENERAL TERMS - Cloud computing, information reliability, open auditing, User revocation.

\section{INTRODUCTION}

With data stockpiling and granting organizations in the cloud, customers can without a doubt change and offer data as a social occasion. To ensure bestowed data uprightness can be checked transparently, customers in the get-together need to process stamps on all the squares in conferred data. Different squares in bestowed data are generally checked by particular customers due to data conformities performed by differing customers. For security reasons, once a customer is revoked from the social affair, the squares which were heretofore stamped by this denied customer must be re-checked by a present customer. The immediate framework, which allows a present customer to download the looking at bit of conferred data and re-sign it in the midst of customer denial, is inefficient in view of the generous size of bestowed data in the cloud. In this paper, we propose a Novel open assessing instrument for the respectability of granted data to beneficial customer denial in mind. In extension, an Open verifier is always prepared to survey the uprightness of conferred data without recouping the entire data from the cloud, paying little heed to the way that some bit of bestowed data has been rechecked by the cloud. Furthermore, our instrument has the limit support group investigating by checking various inspecting assignments at the same time. Trial results exhibit that our framework can in a general sense improve the viability of customer denial.

\section{SYSTEM ARCHITECTURE:}

The building design of proposed framework portrayed in Fig.1. It incorporates three substances: the cloud, people in general verifier, and clients (who offer information as a gathering). The cloud offers information stockpiling and imparting administrations to the gathering. General society verifier, for example, a customer who might want to use cloud information for specific purposes (e.g., seek, processing, information mining, and so forth.) or an outsider inspector (TPA) Who can give check benefits on information respectability, intends to check the trustworthiness of imparted information by means of a test and reaction convention with the cloud. In the gathering, there is one unique client and Various gathering clients. The first client is the first proprietor of information. This unique client makes and shares information with different clients in the gathering through the cloud. Both the first client and gathering clients have the capacity to get to, download and change imparted information. Imparted information is partitioned into various squares. A client in the gathering can adjust a square in imparted information by performing a supplement, erase or upgrade operation on the piece.

In this paper, we accept the cloud itself is semi-trusted, which implies it takes after conventions and does not dirty information respectability effectively as a vindictive enemy, however it may mislead verifiers about the error of imparted information keeping in mind the end goal to spare the notoriety of its information administrations and abstain from losing cash on its Information administrations. Moreover, we additionally accept there is no agreement between the cloud and any client amid the outline of our component. By and large, the inaccuracy of offer information under the above semi trusted model can be presented by equipment/programming disappointments or human lapses happened in the cloud. Considering these variables, clients don't completely believe the cloud with the respectability of imparted information. 
To secure the respectability of imparted information, every square in imparted information is appended to a mark, which is registered by one of the clients in the gathering. In particular, when imparted information is at first made by the first client in the cloud, all the marks on imparted information are registered by the first client. After that, once a client alters a piece, this client likewise needs to sign the altered square with his/her own particular private key. By imparting information among a gathering of clients, distinctive squares may be marked by diverse clients because of changes from distinctive clients.

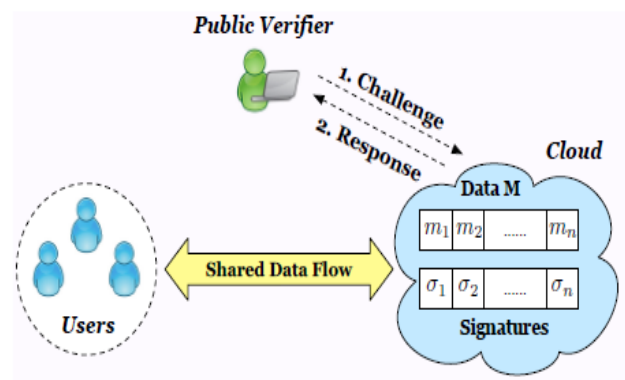

Figure:1.System Architecture

\subsection{User Module:}

\subsubsection{Enlistment: (Registration)}

In this module every client enlist his client subtle elements for utilizing records. Just enlisted client can ready to login in cloud server.

\subsubsection{Document Upload:}

In this module client transfer a piece of records in the cloud with encryption by utilizing his mystery key. This guarantees the records to be shielded from unapproved client.

\subsubsection{Download:}

This module permits the client to download the document utilizing his Mystery key to decode the downloaded information of blocked client and check the information and reupload the piece of record into cloud server with encryption. This guarantees the records to be shielded from unapproved client.

\subsubsection{Reupload:}

This module permit the client to reupload the downloaded documents of blocked client into cloud server with leave the files (i.e) the records is transferred with new mark like new mystery with encryption to shielded the information from unapproved client.

\subsection{Unblock Module:}

This module permits the client to unblock his client account by noting his security inquiry with respect to answer that gave by his at the season of registration. Once the answer is coordinated to the answer of enrollment time answer then just record will be opened.

\subsection{Auditor Module:}

\subsubsection{Record Verification module:}

People in general verifier have the capacity effectively check the uprightness of imparted information. People in general verifier can review the respectability of imparted information without recovering the whole information from the cloud, regardless of the possibility that a few squares in imparted information have been re-marked by the cloud.

\subsubsection{Records View:}

In this module open examiner view the all points of interest of transfer, download, blocked client, re-transfer.

\subsection{Admin Module:}

\subsubsection{View Files:}

In this module public auditor view the all details of upload, download, blocked user, re-upload.

\subsubsection{Block User:}

In this module admin block the misbehave user account to protect the integrity of shared data.

\section{RESULTS AND DISCUSSIONS}

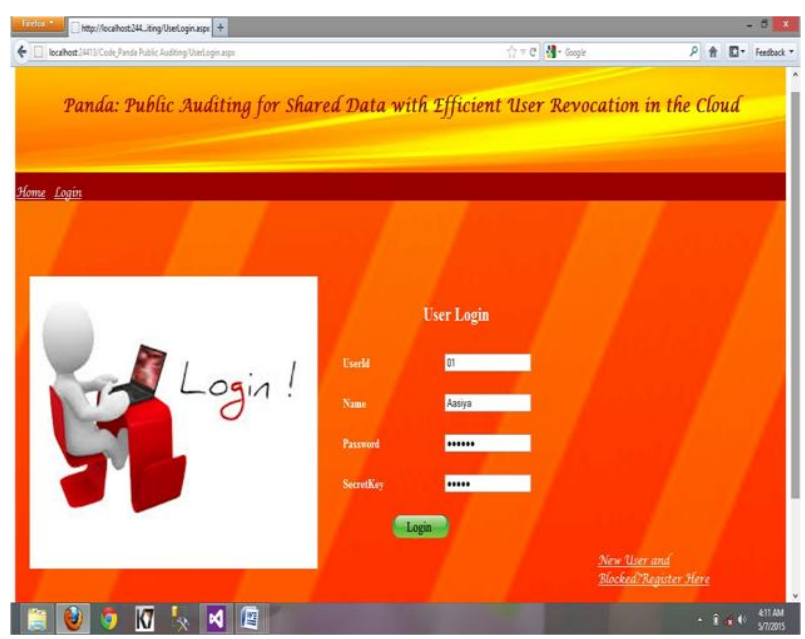

Figure: 3.1.User Login File

The User is the person who shares the data in the group. User can upload the files, download the files and block the user account him/her self.

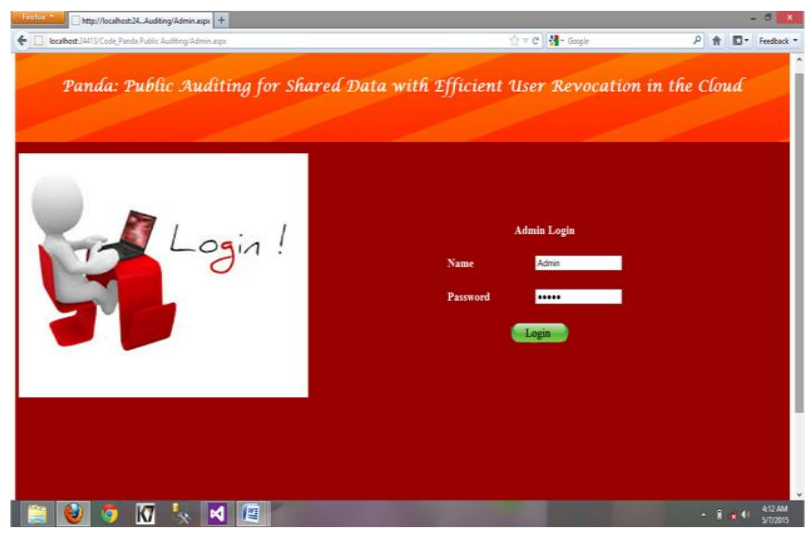

Figure: 3.2.Admin Login File 
The Admin can accept the User's uploaded files and view all uploaded files, downloaded files, modified files and user details.

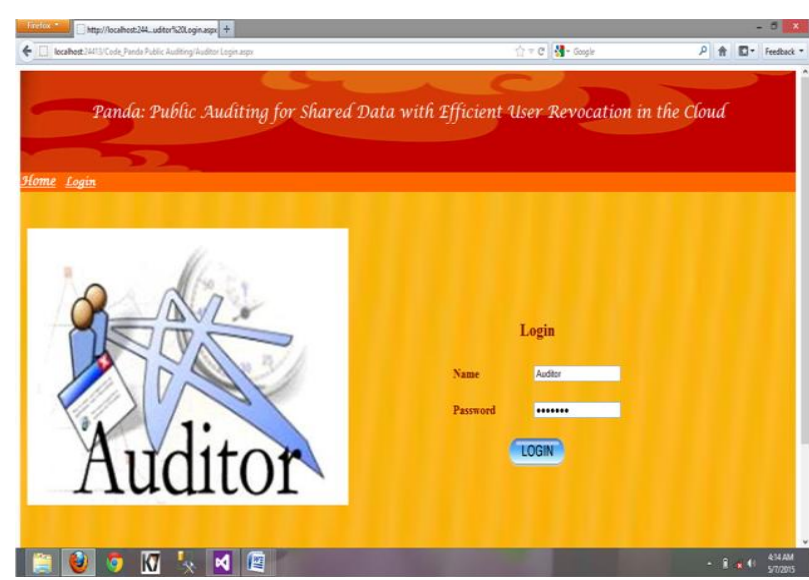

Figure: 3.3.Aditor Login File

The public verifier can audit the integrity of shared data without retrieving the entire data from the cloud, even if some blocks in shared data have been re-signed by the cloud.

\section{CONCLUSION}

In this paper, we proposed another open examining component for imparted information to effective client disavowal in the cloud. At the point when a client in the gathering is renounced, we permit the semi-trusted cloud to re-sign obstructs that were marked by the disavowed client with intermediary re-marks. Trial results demonstrate that the cloud can enhance the proficiency of client disavowal, and existing clients in the gathering can spare a lot of calculation and correspondence assets amid client denial.

\section{ACKNOWLEDGEMENTS}

First and foremost I offer my sincerest gratitude to my college, SVCET and my department of Computer Science and Engineering which has provided the support and equipment I have needed to complete my work. I extend my heartfelt gratitude to my guide, Mr. Nanda Kishore and department of Computer Science, who has supported me throughout our research with their patience and knowledge.

\section{REFERENCES}

[1] Bo yang Wang, Baochun Li, Member, IEEE, and Hui Li, Member, IEEE “ Public Survey For Collective Data With Professional User Revocation In The Cloud "IEEE Transactions on Services Computing, VOL. X, NO. X, XXXX 2014.

[2] P. Mell and T. Grance, "Draft NIST working definition of cloud computing".

[3] C. Wang, Q. Wang, K. Ren, and W. Lou, "Towards Secure and Dependable Storage Services in Cloud
Computing," IEEE Transactions on Services Computing, vol. 5, no. 2, pp. 220-232,2011.

[4] Y. Zhu, G.-J. Ahn, H. Hu, S. S. Yau, H. G. An, and S. Chen, "Dynamic Audit Services for Outsourced Storage in Clouds," IEEE Transactions on Services Computing, accepted.

[5] K. Kiran Kumar, K. Padmaja, P. Radha Krishna, "Automatic Protocol Blocker for Privacy-Preserving Public Auditing in Cloud Computing", International Journal of Computer science and Technology, vol. 3 pp, ISSN. 0976-8491(Online), pp. 936-940, ISSN: 22294333 (Print), March 2012

[6] Jachak K. B., Korde S. K., Ghorpade P. P. and Gagare G. J.,"Homomorphic Authentication with Random Masking Technique Ensuring Privacy \& Security in Cloud Computing", Bioinfo Security Informatics, vol. 2, no. 2, pp. 49-52, ISSN. 2249-9423, 12 April 2012.

[7] J. Yuan and S. Yu, "Proofs of Retrievability with Public Verifiability and Constant Communication Cost in Cloud," in Proceedings of ACM ASIACCS-SCC'13, 2013

[8] H. Shacham and B. Waters, "Compact Proofs of Retrievability,"in the Proceedings of ASIACRYPT 2008. Springer- Verlag, 2008, pp.90-107.

[9] G. Ateniese, R. Burns, R. Curtmola, J. Herring, L. Kissner, Z. Peterson, and D. Song, "Provable Data Possession at Untrusted Stores," in the Proceedings of ACM CCS 2007, 2007, pp. 598-610.

[10] C. Wang, Q. Wang, K. Ren, and W. Lou, "PrivacyPreserving Public Auditing for Data Storage Security in Cloud Computing, "in the Proceedings of IEEE INFOCOM 2010, 2010, pp. 525-533.

[11] N. Cao, S. Yu, Z. Yang, W. Lou, and Y. T. Hou, "LT Codes-based Secure and Reliable Cloud Storage Service," in the Proceedings of IEEE INFOCOM 2012, 2012, pp. 693-701

[12] H. Wang, "Proxy Provable Data Possession in Public Clouds,'IEEE Transactions on Services Computing, accepted.

[13] B. Wang, B. Li, and H. Li, "Oruta: Privacy-Preserving Public Auditing for Shared Data in the Cloud," in the Proceedings of IEEE Cloud 2012, 2012, pp. 295-302.

[14] Q. Wang, C. Wang, J. Li, K. Ren, and W. Lou, "Enabling Public Verifiability and Data Dynamic for Storage Security in Cloud Computing," in the Proceedings of ESORICS 2009. Springer-Verlag, 2009, pp.355-370.

[15] B. Wang, B. Li, and H. Li, "PANDA: Public Auditing for Shared Data with Efficient User Revoation in the Cloud," in the Proceedings of IEEE INFOCOM 2014, 2014, pp.sss 